\title{
Insuficiencia venosa cerebroespinal crónica y esclerosis múltiple: revisión y actualización del tema
}

\author{
Carmen Gasca-Salas, Asier Gómez-Ibáñez
}

Resumen. La etiología de la esclerosis múltiple se desconoce en el momento actual, aunque se acepta el origen inflamatorio autoinmune como el más probable. En la historia de esta enfermedad se propuso una fisiopatología vascular, la cual ha resurgido recientemente a partir de los trabajos de Paolo Zamboni y se ha denominado 'insuficiencia venosa cerebroespinal crónica'. Siguiendo esta hipótesis, Zamboni plantea un tratamiento curativo para la esclerosis múltiple mediante tratamiento endovascular de la vena yugular interna y la vena ácigos. Sin embargo, varios equipos han intentado replicar sus resultados sin conseguirlo. En esta revisión describimos cronológica y objetivamente los estudios tanto de Zamboni como de los intentos posteriores de réplica. Nuestra principal conclusión es que con los resultados disponibles hasta la actualidad debemos ser cautos y no recomendar por el momento este tratamiento a nuestros pacientes de una forma sistemática.

Palabras clave. Angioplastia. Eco-Doppler. Esclerosis múltiple. Hierro. Hipótesis. IVCEC. Venografía.

\section{Introducción}

La esclerosis múltiple (EM) es una enfermedad inmunomediada de evolución crónica, caracterizada por la inflamación, desmielinización y degeneración del sistema nervioso central. La etiología exacta se desconoce, pero hoy día sabemos que la fisiopatología está basada en una interacción compleja de linfocitos $\mathrm{T}$ autorreactivos y células $\mathrm{B}$ que reconocen antígenos de la mielina y atacan al sistema nervioso central [1-3]. Actualmente en España el tratamiento de primera línea en el tipo recurrenteremitente (EMRR) es interferón beta (IFN $\beta$-1b subcutáneo, IFN $\beta$-1a subcutáneo e IFN $\beta$-1a intramuscular), acetato de glatiramero y azatioprina; en caso de evolución rápida y agresiva también está autorizado el uso de natalizumab. En la forma secundariamente progresiva (EMSP), si presenta brotes o actividad inflamatoria en la resonancia magnética (RM), el tratamiento indicado es IFN $\beta$-1b e IFN $\beta$ 1a subcutáneos, con la mitoxantrona como segunda línea en la forma EMSP con brotes; para las primariamente progresivas (EMPP) no hay evidencia de eficacia de ningún tratamiento [4]. Respecto al futuro, las principales novedades terapéuticas recogidas en el último congreso ECTRIMS celebrado en octubre de 2010 son el tratamiento temprano con acetato de glatiramero del síndrome clínico aislado, la terapia combinada en pacientes con EMRR y el uso de anticuerpos monoclonales (alemtuzumab, rituximab, daclizumab, etc.) y agonistas del receptor linfocitario S1P1 linfocitario como el fingolimod [5].

Sin embargo, desde el inicio del estudio de la enfermedad hubo discrepancias entre una hipótesis fisiopatológica inflamatoria, como la admitida actualmente, y otra vascular. Estas diferencias han vuelto a aparecer a raíz de la publicación por parte de Paolo Zamboni -médico italiano especialista en cirugía vascular- de unos trabajos en los que basa la etiopatogenia de la enfermedad en la denominada 'insuficiencia venosa cerebroespinal crónica' (IVCEC). Esta hipótesis ha suscitado cierta controversia en la comunidad científica y ha llevado a la realización de varios trabajos al respecto.

El objetivo de esta revisión es mostrar, de un modo riguroso, los hallazgos tanto de los trabajos de Zamboni en los que explica su hipótesis como los posteriores en los que diferentes autores rebaten sus resultados.

\section{Historia de la hipótesis vascular en la esclerosis múltiple}

La primera vez que se propuso un origen vascular para la EM fue en 1863, por Edward Rindfleisch, tras apreciar vasos congestionados e inflamados en
Departamento de Neurología. Clínica Universidad de Navarra. Pamplona, Navarra, España.

Correspondencia:

Dra. Carmen Gasca Salas. Clínica Universidad de Navarra. Pío XII, 36. E-31008 Pamplona (Navarra).

Fax:

+34948296500

E-mail:

cgasca@unav.es

Agradecimientos:

A la Dra. Purificación de Castro, neuróloga experta en esclerosis múltiple de nuestro centro, por la revisión del artículo.

Aceptado tras revisión externa: 28.04.11.

Cómo citar este artículo: Gasca-Salas C, Gómez-Ibáñez A. Insuficiencia venosa cerebroespinal crónica y esclerosis múltiple: revisión y actualización del tema. Rev Neurol 2011; 53: 555-60.

C 2011 Revista de Neurología 
Tabla I. Criterios de insuficiencia venosa cerebroespinal crónica (traducido de [10]) a.

Reflujo constante en la vena yugular interna o la vena vertebral con la cabeza alineada entre $0^{\circ} y+90^{\circ}$

Reflujo en las venas cerebrales profundas

Estenosis proximal de la vena yugular interna $>50 \%$

en ecografía modo $B$ de alta resolución

Flujo no detectable mediante Doppler en la vena yugular interna 0 en la vena vertebral a pesar de numerosas inspiraciones profundas con la cabeza alineada entre $0^{\circ} y+90^{\circ}$

Incremento negativo de área de sección transversa en la vena yugular interna $^{\mathrm{b}}$

a Los pacientes deben cumplir al menos dos criterios para afirmar que tienen una insuficiencia venosa cerebroespinal crónica; ${ }^{b}$ Aumento de área de sección transversa en la vena yugular interna al pasar de sedestación a decúbito supino.

Tabla II. Tests principales de la Multiple Sclerosis Functional Composite (MSFC).

\section{T25W (Timed 25-Foot Walk)}

Tiempo que tarda el paciente en recorrer una distancia de 25 pies

\section{HPT (9-Hole Peg Test)}

El paciente debe recoger nueve clavijas, insertarlas en los nueve agujeros vacíos y volverlas a sacar en el menor tiempo posible. Esto se hace con cada mano

\section{PASAT (Paced Auditory Serial Addition Test)}

Test numérico en que el paciente tiene que completar una secuencia de dígitos con un orden preestablecido

medio de cada foco de sustancia blanca dañado [6]. Poco después, Jean-Martin Charcot definió la EM como una enfermedad primariamente inflamatoria desmielinizante basada en un trastorno primario de la glía y en la que existen cambios secundarios en los vasos sanguíneos [6]. En 1935, Tracey Putnam sugirió tras estudios con animales que la obstrucción venular es el antecedente inmediato a la formación de las placas, volviendo de nuevo a la hipótesis vascular [7]. En 1965, Torben Fog describió la presencia de una vena central orientada a lo largo del eje de la lesión inflamatoria [8]. Finalmente, en 1986 Franz Alfons Schelling sugirió que el reflujo venoso en el drenaje cerebral y espinal podría explicar algunas características de la EM [9].

\section{Insuficiencia venosa cerebroespinal crónica: la hipótesis vascular del siglo XXI}

La hipótesis vascular de la EM ha permanecido silente y ha quedado desplazada a lo largo de los años por la inflamatoria, base fisiopatológica aceptada en el momento actual. Sin embargo, en 2006 Zamboni retomó esa hipótesis basándose en el paralelismo entre la insuficiencia venosa crónica (IVC) en las extremidades inferiores y la EM, en la conferencia anual organizada por la Royal Society of Medicine (The Big Idea). En dicho foro explicó cómo en la IVC aumentan los depósitos de hierro debido a la salida de eritrocitos por estasis venosa. Este incremento, junto con la extravasación de proteínas intersticiales, representaría el inicio del proceso inflamatorio [10]. Esta idea lo llevó a considerar el estudio de la función venosa cerebral y el papel desempeñado por el hierro en la etiopatogenia de la EM. Inició la investigación mediante la realización de ecografías venosas transcraneales en un grupo de 89 sujetos con diagnóstico de EM y en 60 controles, y encontró más alteraciones hemodinámicas en las venas intracraneales de los pacientes [11].

El siguiente año publicó dos trabajos $[12,13]$ en los que valoró el retorno venoso cerebral a través de las venas extracraneales y propuso una lista de cinco parámetros para su estudio (Tabla I), previamente publicados en un artículo del propio autor [14]. En los resultados del primer trabajo, Zamboni destacó que todos los pacientes y ningún control presentaron al menos dos de estos criterios. De este modo, atribuyó estos hallazgos al diagnóstico de EM con una sensibilidad, especificidad, valor predictivo positivo y valor predictivo negativo para la ecografía extra y transcraneal del 100\% [12]. En el segundo estudio llevó a cabo una venografía selectiva de la vena ácigos y el sistema venoso yugular en 65 pacientes con parámetros ecográficos alterados y 48 controles a los que se les realizó debido a otras patologías no neurológicas (hiperparatiroidismo, varicocele o síndrome de congestión pélvica, síndrome de Cockett, síndrome del desfiladero torácico, colocación de cables de marcapasos o catéteres venosos centrales, estenosis de accesos venosos para hemodiálisis y retirada de filtros de la vena cava). En el estudio venográfico objetivó estenosis, definida como reducción de la luz vascular mayor del $50 \%$ de la vena ácigos, en el $86 \%$ de los pacientes, y de la vena yugular interna (VYI) en el 91\%, sin apreciar estenosis de ninguna vena en los controles. Es importante especificar que dentro de las estenosis incluyó varias malformaciones como anillos, septos, hipoplasias, tortuosidades y membranas. En el trabajo sugiere que 
Tabla III. Publicaciones contrarias a la insuficiencia venosa cerebroespinal crónica como causa de la esclerosis múltiple.

\begin{tabular}{|c|c|c|c|}
\hline & Origen & Métodos & Conclusiones \\
\hline Kahn et al [21] & Estados Unidos & Carta de opinión & $\begin{array}{l}\text { No hay evidencia científica para utilizar métodos } \\
\text { endovasculares en el tratamiento de la EM }\end{array}$ \\
\hline Krogias et al [24] & Alemania & $\begin{array}{l}\text { Estudio neurosonológico } \\
\text { en pacientes con EM }\end{array}$ & $\begin{array}{l}\text { La IVCEC no puede representar } \\
\text { el único factor patogénico en la EM }\end{array}$ \\
\hline Doepp et al [25] & Alemania & $\begin{array}{l}\text { Eco-Doppler extracraneal y transcraneal } \\
\text { en } 56 \text { pacientes y } 20 \text { controles }\end{array}$ & $\begin{array}{l}\text { No hay diferencias significativas en el drenaje } \\
\text { venoso cerebral entre pacientes y controles }\end{array}$ \\
\hline Sundström et al [26] & Austria & $\begin{array}{l}\text { Eco-Doppler extracraneal y transcraneal } \\
\text { en } 21 \text { pacientes y } 20 \text { controles }\end{array}$ & $\begin{array}{l}\text { No existen diferencias significativas entre pacientes } \\
\text { y controles en el flujo o reflujo de la vena yugular } \\
\text { interna ni en el flujo en el acueducto de Silvio }\end{array}$ \\
\hline Wattjes et al [27] & Países Bajos & $\begin{array}{l}\text { Venografía por resonancia magnética } \\
\text { en } 20 \text { pacientes y } 20 \text { controles }\end{array}$ & $\begin{array}{l}\text { Existen alteraciones anatómicas venosas tanto en } \\
\text { pacientes como en controles, pero probablemente } \\
\text { son variantes de la normalidad sin carácter patológico }\end{array}$ \\
\hline Yamout et al [28] & Líbano & $\begin{array}{l}\text { Venografía extracraneal en } 29 \text { pacientes } \\
\text { con EM temprana y } 13 \text { con EM tardía }\end{array}$ & $\begin{array}{l}\text { La estenosis de las venas extracraneales no está } \\
\text { presente en la mayoría de pacientes con EM temprana } \\
\text { y es probablemente un fenómeno secundario }\end{array}$ \\
\hline Baracchini et al [29] & Italia & $\begin{array}{l}\text { Eco-Doppler extracraneal y transcraneal } \\
\text { en } 50 \text { pacientes con EM posible, } \\
60 \text { pacientes con AGT y } 110 \text { controles. } \\
\text { Venografía selectiva si hay IVCEC }\end{array}$ & $\begin{array}{l}\text { No hay relación causa-efecto } \\
\text { entre EM posible e IVCEC }\end{array}$ \\
\hline Mayer et al [30] & Alemania & $\begin{array}{l}\text { Eco-Doppler extracraneal y transcraneal } \\
\text { en } 20 \text { pacientes con EM y } 20 \text { controles. } \\
\text { Triple ciego }\end{array}$ & $\begin{array}{l}\text { No hay evidencia de la presencia } \\
\text { de IVCEC en pacientes con EM }\end{array}$ \\
\hline
\end{tabular}

AGT: amnesia global transitoria; EM: esclerosis múltiple; IVCEC: insuficiencia venosa cerebroespinal crónica.

la localización de éstas constituye un elemento clave del curso clínico de la enfermedad y diferencia cuatro patrones (A, B, C y D) según su localización y número. La presencia de múltiples estenosis graves que afectan a segmentos venosos cerebroespinales extracraneales es lo que denominó IVCEC [13].

Posteriormente, y una vez establecida su hipótesis, publicó un nuevo trabajo con 65 pacientes: 35 tenían un curso de EMRR, 10 de EMPP y 20 de EMSP. A estos pacientes les realizó una angioplastia transluminal simple para dilatar las venas estenosadas. En el seguimiento posterior, los 35 con un curso de EMRR mejoraron en las escalas de calidad de vida y en la MSFC (Multiple Sclerosis Functional Composite) [15,16] (Tabla II) tras 18 meses, y tanto los 10 con curso de EMPP como los 20 con curso de EMSP únicamente mejoraron en las escalas de calidad de vida, sin mejoría en la escala MSCF, y sólo los seis primeros meses [17]. En este punto, el propio Zamboni presentó algunos problemas metodológicos del estudio:
- El estudio no era ciego, ya que todos los pacientes sabían que estaban recibiendo tratamiento.

- Las malformaciones que consideraba como estenosis podían ser variantes de la normalidad.

- Los pacientes del grupo con EMRR mantenían durante el estudio el tratamiento inmunomodulador.

- No existía un protocolo específico para la realización de la RM en estos pacientes.

- El 47\% de los pacientes con estenosis yugular sufrieron reestenosis tras la intervención y no fueron evaluados en los resultados finales.

Estos resultados han llevado a la realización de angioplastia transluminal y colocación de stent en pacientes con EM. El tratamiento es aparentemente seguro y bien tolerado a pesar de algunas complicaciones menores [18] y del fallecimiento conocido de un paciente [19]. Por ello, Zamboni recomienda ser cauteloso hasta que se hayan llevado a cabo más ensayos clínicos, y únicamente realizarlo en este contexto [20]. 
Tabla IV. Consideraciones fisiopatológicas que no apoyan la insuficiencia venosa cerebroespinal crónica en la esclerosis múltiple (traducido de [21]).

Existencia de depósitos de hierro en otras enfermedades neurológicas

La estasis venosa produciría un aumento en

la prevalencia de esclerosis múltiple con la edad

La oclusión venosa ocasionaría mayor prevalencia de infartos cerebrales y edema por incremento de la presión intracraneal

La congestión de la vena yugular interna propiciaría un drenaje alterado de las venas oftálmicas y retinianas, lo que produciría un incremento de hemorragias retinianas no visto en pacientes con esclerosis múltiple

La insuficiencia venosa yugular se ha relacionado con la amnesia global transitoria, entidad no más frecuente en pacientes con esclerosis múltiple que en la población general

No existen casos descritos de esclerosis múltiple tras disección radical cervical, en la que se extirpa la vena yugular interna

Se ha descrito un incremento de la presión venosa cerebral en patologías que no se han asociado con esclerosis múltiple (trombosis venosa cerebral, hipertensión intracraneal idiopática, hipertensión pulmonar y enfermedad pulmonar obstructiva crónica)

\section{Controversias respecto a la insuficiencia venosa cerebroespinal crónica}

A raíz de las investigaciones de Zamboni, en 2010 empezaron a aparecer trabajos que intentaban replicar sus hallazgos (Tabla III). En marzo, el equipo de Khan publicó un artículo señalando la falta de consistencia de estos hallazgos, tanto desde un punto de vista metodológico como fisiopatológico. Respecto al primero, subrayó las limitaciones ya reconocidas por el propio Zamboni y comentadas previamente. En cuanto a la fisiopatología, detalló las consecuencias sobre el sistema nervioso central y visual derivadas de la alteración en el sistema venoso propuesto (Tabla IV). Los autores concluyeron que, actualmente, los procedimientos endovasculares invasivos en pacientes con EM deberían evitarse hasta que haya una evidencia concluyente que justifique su indicación [21].

Un mes después, en el congreso de la Asociación Americana de Neurología, Zamboni junto con Zivadinov y otros autores mostraron cómo los hallazgos de IVCEC aparecían también en algunos controles sanos y no sólo en pacientes con EM [22]. En ese mismo mes de abril publicaron un estudio mediante eco-Doppler, venografía por RM (VRM) y venografía selectiva en diez pacientes, y únicamente eco-Doppler y VRM en siete controles. Todos los pacientes y ningún control cumplían los criterios ecográficos de IVCEC. Además, vieron que los hallazgos del eco-Doppler y la VRM no eran superponibles en siete pacientes y cuatro controles. La conclusión fue que el uso de la VRM para el diagnóstico de IVCEC en la EM tiene un valor limitado [23].

Dos meses después, un grupo alemán publicó un estudio en el que intentó reproducir estos hallazgos, pero sólo el $20 \%$ de sus pacientes cumplía los criterios neurosonológicos de IVCEC. Por tanto, concluyó que la IVCEC no puede representar el factor patogénico exclusivo en la EM [24]. En agosto, otro grupo diferente, también de Alemania, no evidenció mediante eco-Doppler diferencias significativas entre pacientes y controles, y sugirió que el drenaje venoso cerebral en la EM no estaba restringido [25]. Además, describieron algunas diferencias metodológicas respecto al estudio de Zamboni, como la presencia de falsos positivos en la estenosis de la VYI por su fácil compresión o las variaciones fisiológicas del diámetro de esta vena según la posición del sujeto y la presión torácica y central [25]. Un grupo sueco, en esas mismas fechas, estudió mediante RM el flujo sanguíneo cerebral, y concluyó que éste era ligeramente menor en pacientes con EM, probablemente secundario a atrofia cerebral. En controles, las diferencias respecto al flujo no fueron estadísticamente significativas. Tampoco hubo diferencias significativas en el flujo de la VYI y el número de pacientes con reflujo de la VYI fue similar en ambos grupos [26].

En octubre aparecieron otros dos trabajos: uno holandés mostró que en 10 de 20 pacientes y 8 de 20 controles existían alteraciones anatómicas venosas medidas por VRM, y afirmó que las alteraciones venosas en estos sujetos podrían ser variantes de la normalidad [27]; y otro equipo libanés planteó que la estenosis venosa extracraneal es una causa improbable de EM tras comprobar que sólo 19 de 42 pacientes, la mayoría de larga evolución, presentaban estenosis venosa extracraneal. Por ello, sugirieron que la estenosis podría ser un fenómeno secundario a atrofia del sistema nervioso central [28].

Ya en 2011, un estudio de 50 pacientes con síndrome clínico aislado y diseminación en el espacio, con diagnóstico de esclerosis múltiple posible (EMp), 60 pacientes con amnesia global transitoria y 110 controles evidenció que sólo 8 pacientes con EMp cumplían los criterios diagnósticos de IVCEC; siete de ellos fueron sometidos a venografía selectiva y ninguno mostró anomalías venosas [29]. Finalmente, el último trabajo corresponde a un grupo alemán que ha realizado un estudio triple ciego (ecografista, analizador de datos y estadístico) con 20 pacientes y 20 controles en el que únicamente un control 
cumple al menos dos criterios de IVCEC, mientras que ningún paciente presentaba alteraciones [30].

Por otra parte, se sabe de la presencia de depósitos de hierro en las placas de EM [31-33], al igual que en patologías primariamente degenerativas como la enfermedad de Alzheimer o la enfermedad de Parkinson [34]. Zamboni, en un trabajo de revisión publicado en 2009, sugirió una sobrecarga de estos depósitos de hierro en la EM como consecuencia de la alteración del retorno venoso cerebral y del drenaje venoso anómalo crónico [35]. Para ello, se basó en la hipótesis de la IVCEC, la histología de los espacios perivenosos dentro de la placa de EM [33] y técnicas de RM avanzada [36,37]. En relación con esta idea, un estudio publicado en 2010 reveló que, mientras los niveles de ferritina en el líquido cefalorraquídeo estaban elevados en pacientes con patologías con incremento del depósito de hierro (siderosis superficial y hemorragia subaracnoidea), en pacientes con EM estos niveles estaban dentro de la normalidad (hasta $12 \mathrm{ng} / \mathrm{mL}$ ) [38].

\section{Conclusión}

Actualmente podemos afirmar que la mayoría de los estudios realizados por equipos diferentes al de Zamboni siembran dudas razonables sobre la existencia de la IVCEC y su participación en la fisiopatología de la EM. Es motivo de alegría que algún paciente haya podido mejorar con el tratamiento endovascular, pero ello no implica ni que todos vayan a mejorar ni que la mejoría sea consecuencia de la intervención. Además, dada la ausencia de un tratamiento curativo para la EM, hay que tener en cuenta que algunos pacientes pueden ser muy susceptibles de ser convencidos con facilidad y aceptar cualquier tratamiento.

Finalmente, pensamos que debemos estar atentos a los resultados de futuros ensayos clínicos, pero aún es pronto para recomendar de un modo sistemático este tratamiento en nuestros pacientes.

\section{Bibliografía}

1. Frohman EM, Racke MK, Raine CS. Multiple sclerosis -the plaque and its pathogenesis. N Engl J Med 2006; 354: 942-55.

2. McFarland HF, Martin R. Multiple sclerosis: a complicated picture of autoimmunity. Nat Inmunol 2007; 9: 913-9.

3. Fernández-Fernández O, Álvarez-Cermeño JC, ArbizuUrdiain T, Arroyo-González R, Arnal-García C, CasanovaEstruch B, et al; Grupo Post-ECTRIMS. Revisión de las novedades presentadas en el XXVI Congreso del Comité Europeo para el Tratamiento e Investigación en Esclerosis Múltiple (ECTRIMS) (I). Rev Neurol 2011; 52: 227-38.

4. García-Merino A, Fernández O, Montalbán X, De Andrés C, Arbizu T. Documento de consenso de la Sociedad Española de Neurología sobre el uso de medicamentos en esclerosis múltiple: escalado terapéutico. Neurología 2010; 25: 378-90.

5. Fernández-Fernández O, Álvarez-Cermeño JC, ArbizuUrdiain T, Arroyo-González R, Arnal-García C, CasanovaEstruch B, et al; Grupo Post-ECTRIMS. Revisión de las novedades presentadas en el XXVI Congreso del Comité Europeo para el Tratamiento e Investigación en Esclerosis Múltiple (ECTRIMS) (II). Rev Neurol 2011; 52: 289-99.

6. Compston A, McAlpine D. McAlpine's multiple sclerosis. Philadelphia: Churchill-Livingstone Elsevier; 2006.

7. Putnam TJ. Studies in multiple sclerosis: 'encephalitis' and sclerotic plaques produced by venular obstruction. Arch Neurol Psychiatry 1935; 33: 929-40.

8. Fog T. The topography of plaques in multiple sclerosis with special reference to cerebral plaques. Acta Neurol Scand Suppl 1965; 15: 1-161.

9. Schelling F. Damaging venous reflux into the skull or spine: relevance to multiple sclerosis. Med Hypotheses 1986; 21 : 141-8.

10. Zamboni P. The big idea: iron-dependent inflammation in venous disease and proposed parallels in multiple sclerosis. J R Soc Med 2006; 99: 589-93.

11. Zamboni P, Menegatti E, Bartolomei I, Galeotti R, Malagoni AM, Tacconi G, et al. Intracranial venous haemodynamics in multiple sclerosis. Curr Neurovasc Res 2007; 4: 252-8.

12. Zamboni P, Menegatti E, Galeotti R, Malagoni AM, Tacconi G, Dall'Ara S, et al. The value of cerebral Doppler venous haemodynamics in the assessment of multiple sclerosis. J Neurol Sci 2009; 282: 21-7.

13. Zamboni P, Galeotti R, Menegatti E, Malagoni AM, Tacconi G, Dall'Ara S, et al. Chronic cerebrospinal insufficiency in patients with multiple sclerosis. J Neurol Neurosurg Psychiatry 2009; 80: 392-9.

14. Menegatti E, Zamboni P. Doppler haemodynamics of cerebral venous return. Curr Neurovasc Res 2008; 5: 260-5.

15. Cutter GR, Baier ML, Rudick RA, Cookfair DL, Fischer JS, Petkau J, et al. Development of a multiple sclerosis functional composite as a clinical trial outcome measure. Brain 1999; 122: 871-82.

16. Fischer JS, Rudick RA, Cutter GR, Reingold SC. The Multiple Sclerosis Functional Composite Measure (MSFC): an integrated approach to MS clinical outcome assessment. National MS Society Clinical Outcomes Assessment Task Force. Mult Scler 1999; 5: 244-50.

17. Zamboni P, Galeotti R, Menegatti E, Malagoni AM, Gianesini $\mathrm{S}$, Bartolomei I, et al. A prospective open-label study of endovascular treatment of chronic cerebrospinal venous insufficiency. J Vasc Surg 2009; 50: 1348-58.

18. Ludyga T, Kazibudzki M, Simka M, Hartel M, Swierad M, Piegza J, et al. Endovascular treatment for chronic cerebrospinal venous insufficiency: is the procedure safe? Phlebology 2010; 25: 286-95.

19. The Wall Street Journal. MS program halted amid controversy. URL: http://online.wsj.com/article/SB100014240527487042 $11704575140313904335240 . h t m l$ mod=WSJ_hpp_MIDDLTopStories. [06.04.2011].

20. MS News Today. Hot off the ECTRIMS Press: Zamboni cautions further studies needed on CCSVI. URL: http:// www.msnewstoday.com/hot-off-the-ectrims-press-zambonicautions-further-studies-needed-on-ccsvi. [06.04.2011].

21. Khan O, Filippi M, Freedman MS, Barkhof F, Dore-Duffy P, Lassmann $\mathrm{H}$, et al. Chronic cerebrospinal venous insufficiency and multiple sclerosis. Ann Neurol 2010; 67: 286-90.

22. Zivadinov R, Marr K, Ramanathan M, Zamboni P, Benedict RRHB, Cutter G, et al. Combined transcranial and extracranial venous Doppler evaluation (CTEVD STUDY). Description of the design and interim results of epidemiological study of the prevalence of chronic cerebrospinal venous insufficiency in MS and related diseases. Poster Session presented at: 62nd AAN Annual Meeting; 2010 April 15; Toronto, Canada [P06.144]. Neurology 2010; 74: A545.

23. Hojnacki D, Zamboni P, López-Soriano A, Galleotti R, Menegatti E, Weinstock-Guttman B, et al. Use of neck 
magnetic resonance venography, Doppler sonography and selective venography for diagnosis of chronic cerebrospinal venous insufficiency: a pilot study in multiple sclerosis patients and healthy controls. Int Angiol 2010; 29: 127-39.

24. Krogias C, Schröder A, Wiendi H, Hohlfeld R, Gold R. 'Chronic cerebrospinal venous insufficiency' and multiple sclerosis: critical analysis and first observation in an unselected cohort of MS patients. Nervenarzt 2010; 81: 740-6.

25. Doepp F, Paul F, Valdueza JM, Schmierer K, Schreiber SJ. Cerebrocervical venous congestion in patients with multiple sclerosis. Ann Neurol 2010; 68: 173-83.

26. Sundström P, Wåhlin A, Ambarki K, Birgander R, Eklund A, Malm J. Venous and cerebrospinal fluid flow in multiple sclerosis: a case-control study. Ann Neurol 2010; 68: 255-9.

27. Wattjes MP, Van Oosten BW, De Graaf WL, Seewann A, Bot JC, Van den Berg R, et al. No association of abnormal cranial venous drainage with multiple sclerosis: a magnetic resonance venography and flow-quantification study. J Neurol Neurosurg Psychiatry 2011; 82: 429-35.

28. Yamout B, Herlopian A, Issa Z, Habib RH, Fawaz A, Salame J, et al. Extracranial venous stenosis is an unlikely cause of multiple sclerosis. Mult Scler 2010; 16: 1341-8.

29. Baracchini C, Perini P, Calabrese M, Causin F, Rinaldi F, Gallo P. No evidence of chronic cerebrospinal venous insufficiency at multiple sclerosis onset. Ann Neurol 2011; 69: 90-9.

30. Mayer CA, Pfeilschifter W, Lorenz MW, Nedelmann M,
Bechmann I, Steinmetz H, et al. The perfect crime? CCSVI not leaving a trace in MS. J Neurol Neurosurg Psychiatry 2011; 82: 436-40

31. Craelius W, Migdal MW, Luessenhop CP, Sugar A, Mihalakis I. Iron deposits surrounding multiple sclerosis plaques. Arch Pathol Lab Med 1982; 106: 397-9.

32. Walton JC, Kaufmann JC. Iron deposits and multiple sclerosis. Arch Pathol Lab Med 1984; 108: 755-6.

33. Adams CW. Perivascular iron deposition and other vascular damage in multiple sclerosis. J Neurol Neurosurg Psychiatry 1988; 51: 260-5.

34. Benarroch EE. Brain iron homeostasis and neurodegenerative disease. Neurology 2009; 72: 1436-40.

35. Singh AV, Zamboni P. Anomalous venous flow and iron deposition in multiple sclerosis. J Cereb Blood Flow Metab 2009; 29: 1867-8.

36. Haacke EM, Cheng NY, House MJ, Liu Q, Neelavalli J, Ogg RJ, et al. Imaging iron stores in the brain using magnetic resonance imaging. Magn Reson Imagin 2005; 23: 1-25.

37. Tjoa CW, Benedict RH, Weinstock-Guttman B, Fabiano AJ, Bakshi R. MRI T2 hypointensity of the dentate nucleus is related to ambulatory impairment in multiple sclerosis. J Neurol Sci 2005; 234: 17-24.

38. Worthington V, Killestein J, Eikelenboom MJ, Teunissen CE, Barkhof F, Polman CH, et al. Normal CSF ferritin levels in MS suggest against etiologic role of chronic venous insufficiency. Neurology 2010; 75: 1617-22.

\section{Chronic cerebrospinal venous insufficiency and multiple sclerosis: a review and update of the subject}

Summary. The aetiology of multiple sclerosis remains unknown at the present time, although the most likely explanation is that it has an autoimmune inflammatory origin. During the history of this disease a vascular pathophysiology was once proposed, and it has recently re-emerged as a result of the work by Paolo Zamboni with the name of 'chronic cerebrospinal venous insufficiency'. Following this hypothesis, Zamboni puts forward a curative treatment for multiple sclerosis by means of endovascular treatment of the internal jugular vein and the azygos vein. However, several teams have attempted to replicate his findings without success. In this review, we offer a chronological description of the studies carried out by Zamboni and the later attempts to replicate his work. Our main conclusion is that, given the results we currently have available, we should be cautious and, for the time being, it would be advisable not to recommend the systematic use of this treatment for our patients.

Key words. Angioplasty. CCSVI. Doppler ultrasound. Hypothesis. Iron. Multiple sclerosis. Venography. 